Développement des territoires et communication : politiques et pratiques à l'œuvre

\title{
De la « vietcommunication » comme processus d'insertion et de communication vietnamiens
}

On "Viet-communication" as a Vietnamese Process of Insertion and Communication

\section{Sandrine Basilico}

\section{OpenEdition} Journals

Édition électronique

URL : http://journals.openedition.org/edc/105

DOI : 10.4000/edc.105

ISSN : 2101-0366

Éditeur

Université Lille-3

Édition imprimée

Date de publication : 1 décembre 2003

ISSN : $1270-6841$

Référence électronique

Sandrine Basilico, «De la « vietcommunication » comme processus d'insertion et de communication vietnamiens », Études de communication [En ligne], 26 | 2003, mis en ligne le 10 octobre 2008, consulté le 19 avril 2019. URL : http://journals.openedition.org/edc/105; DOI : 10.4000/edc.105

Ce document a été généré automatiquement le 19 avril 2019

(c) Tous droits réservés 


\section{De la « vietcommunication » comme processus d'insertion et de communication vietnamiens}

On "Viet-communication" as a Vietnamese Process of Insertion and Communication

Sandrine Basilico

1 Pays multi-ethnique, le Viêt-Nam se compose, en dehors de l'ethnie majoritaire Viêt (ou Kinh) ${ }^{1}$, de 54 ethnies minoritaires vivant sur les hauts plateaux du pays. Celles-ci possèdent des coutumes, un genre de vie qui leur sont propres. Ainsi, pour bon nombre d'entre elles, l'habitation est sur pilotis, contrairement aux Viêt dont les maisons sont construites de plain pied; divers costumes aux couleurs chatoyantes les différencient les unes des autres; des coutumes et croyances propres les animent ${ }^{2}$. Bien longtemps, ces minorités sont restées oubliées, en marge de tout développement économique. Mais depuis 1995, et surtout 1997, elles se mettent à consommer toute sorte d'objets occidentalisés ${ }^{3}$, copiant le modèle Viêt pour elles synonyme de progrès.

Il s'agit de la «Viêtcommunication », concept que nous avons développé dans notre étude doctorale, en tant que processus d'insertion des ethnies Thaï et Muong dans l'État-Nation viêtnamien en développement, plus principalement au travers de l'étude des modifications de leur habitation, tant au niveau structurel qu'architectural. C'est-à-dire à travers l'étude des objets échangés, essentiellement occidentaux, que l'on retrouve désormais au sein des habitations des ethnies minoritaires (appelées aussi «montagnards »).

3 Nous sommes allée nous rendre compte de ces changements sur place lors de deux voyages effectués au Viêt-Nam de septembre à octobre 1995 puis 1997, plus principalement dans la région de Hoa Binh ${ }^{4}$. Notre premier séjour de 1995 était une prise de contact avec la vie des minorités, au cours de laquelle nous avons constaté des différences importantes entre leur vie actuelle et la plupart des écrits qui leur sont consacrés. Notre séjour de 1997, quant à lui, consistait à vérifier cette impression, par le 
truchement d'un questionnaire, d'entretiens non-directifs mais aussi par l'observation participante.

4 C'est donc cette période de 1995-1997, pour laquelle nous disposons d'un terrain accompagné de nombreuses données, qui fera l'objet du présent article.

5 Ce concept nouveau cherche donc à examiner à la fois la manière et le contenu de la communication entre minorités et majorité à travers l'habitation et ses changements. Formé des mots "viêt» et "communication", il introduit un aspect dynamique, complexe aussi, puisque s'étageant à tous les stades de la société (culture, économie, politique). En effet, l'introduction de la notion de communication tend à laisser supposer que l'institutionnalisation du comportement des minorités ne s'exerce pas dans le vide, mais dans un complexe social donné, donc systémique. Ce complexe social, c'est le modèle viêt. Par ce concept nous entendons des changements plus ou moins radicaux au sein des ethnies minoritaires, mais aussi au sein de la majorité qui se voit «enrichie » d'un ou de plusieurs nouveaux groupes, dont la structure était au départ fort différente. Ces changements sont le fruit des politiques menées par le Parti d'une part et par l'éclosion de l'économie de marché, d'autre part.

\section{Les politiques étatiques}

6 Aux frontières de la Chine, du Laos et du Cambodge, on comprend que les minorités occupent une place stratégique. Courtisées d'une part par les colons français, d'autre part par les révolutionnaires - car " qui tient les plateaux, tient l'Indochine ${ }^{5}$, elles n'en sont pas moins restées «étrangères [...] par leur mode d'organisation et leur stade de développement à celles des Viêtnamiens des plaines» (Rousset, 1978, p. 196). C'est ainsi que les habitants des plaines, s'ils ne persistent plus à penser que les montagnards sont des "sauvages", continuent néanmoins de faire la différence entre les gens « d'en haut », « du milieu » et «du bas »...

7 Dans l'histoire du Viêt-Nam, seuls Hô Chi Minh et les révolutionnaires Viêtnamiens ont eu une longue expérience de vie commune avec les minorités des montagnes (1930-1954). Dès 1929, le mouvement révolutionnaire s'est implanté dans la province de Cao Bang, pays des minorités.

8 Celles-ci acquièrent alors une dimension toute politique :

Le problème des ethnies est d'abord politique avant de devenir un sujet de recherches scientifiques. Dans notre pays, des peuples frères ont mené une lutte commune durant de nombreux siècles, ont élaboré ensemble une tradition d'union, de solidarité, d'entr'aide pour vivre et se développer, pour constituer un État et le défendre contre toute agression étrangère (Len Hao, 1971, p. 10).

9 Ici, tout est dit sur les principes politiques du Parti, les ethnies étant considérées comme un problème politique à savoir que l'idéologie communiste s'y applique tout entière.

10 L'État va alors mettre en œuvre toute une série de politiques visant à liquider l'écart entre la plaine et la montagne.

\section{Politique économique}

11 Les régions montagneuses ont, semble-t-il, de grandes potentialités économiques : 
Pourvues de vastes étendues de terre, de forêts, de sources d'eau, d'engrais, de bêtes de somme et jouissant par ailleurs d'un climat favorable, elles ont toutes les conditions requises pour développer une agriculture diversifiée : plantes vivrières et industrielles, élevage, sylviculture. Elles recèlent aussi de nombreux minerais précieux: fer, étain, lignite, plomb, zinc, apatite, bauxite, manganèse... Leur potentiel hydroélectrique est considérable (Quang Canh, 1967, p. 92).

La main d'œuvre supplémentaire qui vient du delta en vue de participer au développement économique et culturel des régions montagneuses a pour leitmotiv de les aider à « rattraper le peuple majoritaire du delta et réaliser l'égalité absolue entre les nationalités »(Quang Canh, 1967, p. 92). Il va sans dire que ces "gens du pays», qui ont subi l'influence des ethnies ont eux aussi apporté un mode de vie assez différent, celui des Viêt. On voit ainsi fleurir en bordure de route près des villages Thaï ou Muong, de nombreuses maisons construites à même le sol, habitées par des Kinh, ne se mêlant pas vraiment à la vie des montagnards et restant aux abords des villages, sur des axes stratégiques leur permettant de se livrer au commerce. Gardant leur mode d'habitation, ils sont un facteur d'influence important.

13 La fonction économique ouvre donc ou devrait ouvrir la tribu hors de la Nation.

\section{Politique d'hygiène}

Depuis plus de 10 ans, une politique d'hygiène est menée, afin de tenter d'enrayer les maladies auxquelles le Viêt-Nam est confronté. Ce qui touche tout particulièrement l'habitation. En effet, l'objectif du gouvernement était d'éduquer les minorités pour construire quelques annexes et notamment des WC situés hors de l'habitation principale ainsi qu'un logement pour le bétail en dehors du pilotis.

Une seconde mesure a donné des résultats plus mitigés puisqu'elle touche justement aux croyances les plus profondes des montagnards, particulièrement des Muong. Il s'agit du foyer, qu'il est recommandé de déplacer de l'habitation principale à la cuisine afin d'éviter tout risque d'incendie. Si les Thaï ont bien suivi cette recommandation, il n'en va pas de même pour les Muong chez qui le génie du foyer a une importance capitale ${ }^{6}$.

16 Ainsi, la politique menée en matière d'hygiène est à la source de nombreuses modifications de l'habitat, en particulier dans la construction des annexes et le déplacement du foyer. Or, l'habitat étant un "ensemble socialement organisé intégrant la vie familiale et individuelle dans les manifestations de la vie sociale et collective ${ }^{7}$, toute modification entraîne inévitablement des bouleversements culturels. C'est le cas, par exemple, pour le foyer, et nous pouvons nous demander si, dans l'esprit des Thaï, le génie du foyer aurait disparu ou se serait déplacé et aurait, par la même occasion, changé de rôle.

\section{Politique d'éducation}

17 L'enseignement et l'éducation sont au cœur des stratégies de développement de la RSV : il s'agit de :

Mettre en œuvre tous les facteurs éducatifs pour former les futures générations de Viêtnamiens nouveaux socialistes (Fourniau, 1985, p. 235). Ainsi, illettrés et superstitieux sous l'ancien régime, nos montagnards sont devenus des hommes dotés d'instruction et ouverts à la culture. Le moins qu'on puisse dire, c'est qu'ils 
ont fait un véritable bond dans le domaine de l'enseignement (Nhat Hung, 1967, p. 117).

18 Par le terme «nos ", le Parti montre qu'il considère que les minorités font partie intégrante de l'État-Nation viêtnamien. Il s'en dégage également un certain paternalisme qui n'est pas sans rappeler l'éthique des mandarins de jadis : " père et mère du peuple ». La politique d'éducation est sans doute la plus importante car elle entraîne, dès le plus jeune âge, une modification du type de réflexion, une manière différente de voir les choses (particulièrement envers les us et coutumes jugées « arriérées » par les Kinh). Les enseignants Viêt ne peuvent être sans influence, de même que les modifications du programme scolaire, où l'on apprend que l'on fait partie d'une nation, voire d'une aire géographique, l'Asie du sud-est, bref où l'on se sent moins isolé des autres peuples. En effet, la politique d'éducation, par rapport à toute autre politique, introduit une autre dimension : le développement chez tout être d'appartenir à un monde plus large.

\section{Politique culturelle}

20 La politique culturelle concernant les mœurs et coutumes des minorités est à cet égard édifiante : celles-ci peuvent avoir un aspect négatif qu'il faut, selon le Parti, modifier ou éliminer, mais elles comportent aussi des traits positifs qu'il convient de préserver et développer car à qui se sent peu attaché à sa culture nationale, il peut très facilement se couper du peuple et, dans certaines circonstances, abandonner même sa patrie. Ainsi, l'ancien patrimoine culturel et artistique des minorités a été peu à peu redécouvert et, " après avoir été poli et repoli, imprimé ou monté en scène est livré à l'appréciation des masses " (Nhat Hung, 1967, p. 59). Le changement d'orientation opéré entraîne, selon nous, des modifications dans l'évolution culturelle, littéraire et artistique des ethnies. Pour mieux assimiler, dans le futur, les montagnards... L'architecture de chaque maison, l'aménagement de chaque cour, chaque allée doivent cadrer avec le nouveau régime, l'économie nouvelle, la culture nouvelle et l'homme nouveau.

21 Les traditions doivent être préservées. Mais que reste-t-il de traditionnel dans une maison construite avec des matériaux modernes ? Et, dans une certaine mesure, pourquoi tant d'acharnement à préserver une culture alors que l'objectif est de «liquider l'écart entre la plaine et la montagne » ? Pourquoi ne pas laisser l'évolution suivre son cours sans intervenir? Que pourrait cacher ou voudrait éviter une telle politique d'intégration par la préservation? Il s'agit bien d'intégration, dont le but ultime est l'assimilation des ethnies minoritaires dont l'aspect premier est le développement des valeurs et aspirations compatibles avec les valeurs ou attitudes de la société d'accueil et susceptibles de trouver leur réalisation en son sein.

\section{Politique linguistique}

La politique linguistique viêtnamienne fait partie intégrante de la politique des nationalités, elle-même basée sur une égalité de principe entre toutes ethnies de la Nation (Agence de Cooperation culturelle et technique, 1984, p. 103).

A ce jour, seulement 8 minorités linguistiques ont une écriture et une langue enseignées à l'école. Seulement 8 ethnies sur 54 , ont été jugées possédant une langue suffisamment «riche». Rien de plus subjectif... Il semblerait que le Parti, derrière son approche progressiste et ses principes politiques, garde une certaine subjectivité, agissant selon les mêmes critères que les Empereurs d'autrefois, en délimitant les champs du « sauvage » et 
du «civilisé ». On peut penser que l'État se construit en fait stratégiquement, avec de nombreux replis car aujourd'hui il n'est pas de bon ton d'écraser les ethnies.

\section{Synthèse} la présence Kinh, dont le commerce est l'un des principaux attributs. Ils vont donc dans les régions montagneuses, parfois même y habitent pour les marchés les plus importants dont l'ouverture est journalière, et proposent toutes sortes de produits allant des légumes au produit le plus moderne. tant au niveau national qu'international. Les ethnies sont ainsi visitées par toutes sortes de personnes, que ce soit des Viêt, des Chinois ou, à un degré moindre, des Occidentaux. Elles ont alors tenté de sauvegarder ce qu'on attend d'elles, c'est-à-dire leur artisanat (instruments de musique, vêtements tissés vendus en guise de souvenir), leurs coutumes (spectacles de danses traditionnelles) ainsi que leur genre de vie. Il y a là une demande " exotique ", au sens où l'entendait Segalen. Le problème, c'est que l'ethnie tend à la modernisation ; le tourisme la renvoie à ses traditions, et peut être, les vivifie-t-il !

\section{Développement du niveau de vie et du genre de vie}

Cette nouvelle activité a considérablement augmenté le niveau de vie des populations qui s'y adonnent, leur permettant aujourd'hui d'acheter des produits modernes auxquels elles n'avaient pas accès faute de moyens ${ }^{10}$. 


\begin{tabular}{|c|c|c|}
\hline & NEUF & OCCASION \\
\hline Télévision & $152 €$ & $76 €$ \\
\hline Chaine hi-fi & $304 \in$ & $122 \epsilon$ \\
\hline Radio & $15 \in$ & $7.5 e$ \\
\hline Réfrigérateur & $304 \in$ & $152 €$ \\
\hline Magnétoscope & $304 \in$ & $122 \epsilon$ \\
\hline Mobylette & $914 \in$ & $609 \in$ \\
\hline Ventilateur & $45.5 €$ & $15 e$ \\
\hline
\end{tabular}

Aujourd'hui, même les produits artisanaux sont manufacturés :

L'économie montagnarde a fortement augmenté. Les maisons sont encore largement construites avec des produits locaux bien que les tuiles soient souvent achetées chez un producteur spécialisé. Les femmes portent encore le costume traditionnel mais il sort souvent d'une manufacture [...]. Les manteaux en plastique ont remplacé le manteau de feuilles de latanier et les lampes torches, avec une demande insatiable de piles, ont remplacé les torches de bambou. Le processus décrit ici fait baisser l'autonomie locale et accroître la dépendance à l'égard des forces et institutions extra locales (Rambo, 1997, p. 24).

\section{Développement du sentiment d'appartenance}

Le fait de se développer économiquement, d'utiliser des produits manufacturés fabriqués par les Viêt, mais aussi les nombreuses guerres connues, les relations touristiques et commerciales avec l'ethnie majoritaire, l'intérêt réciproque que se porte chaque ethnie, la politique d'intégration menée par le gouvernement ${ }^{11}$, la migration de nombreux Viêt dans les régions montagneuses, tout ceci ne peut, nous semble-t-il, que favoriser la conscience d'appartenir à l'entité nationale viêtnamienne, autrement dit, à une entité élargie. Mais plus que cela, c'est peut être l'acquisition d'objets occidentalisés qui en serait le miroir, car synonymes de modèle de développement pour les montagnards, modèle qui n'est autre que Viêt. Nous pensons notamment à la forte influence de la télévision :

Les radios, télévisions, et autres objets électroniques sont devenus une priorité pour la plupart des gens. Peu chères, les batteries venant de Chine permettent d'utiliser la télévision presque partout. Aussi, l'expansion de l'aire couverte par la télévision crée un mixage et détériore la préservation culturelle, offre plus d'opportunité pour des travaux novateurs. Le manque de travailleurs et de moyens de transport peut être transcendé par une production appropriée des programmes télévisés (Rambo, 1997, p. 24).

C'est ainsi que de nombreux $"$ soap $~^{12}$, fort prisés des montagnards, apparaissent. La plupart montrent de riches familles Viêt évoluant dans de vastes maisons en Ao Dai ${ }^{13}$, d'autres nous présentent la jeunesse Viêtnamienne, avec ses jeans, ses motos, ses chewing-gum et ses cigarettes. Bref, tout ce qui peut donner envie de partager ce mode de vie aux montagnards... «Dans les années 80, l'extension agraire a réussi grâce à un 'soap opera' produit par l'université de Can Tho » (Rambo, 1997, p. 24). 
une tendance à la croissance parmi les minorités pour évaluer leur propre style de vie par rapport aux standards nationaux idéalisés auxquels elles sont exposées par les mass media, particulièrement la télévision. Elles se sentent pauvres par rapport à l'affluence de produits qui arrivent devant leurs yeux. Pourtant, elles vivent dans de plus grandes maisons, ont des conditions environnementales agréables, sont moins soumises à la routine que les gens de la plaine. Mais c'est ce que font croire les «soap opera", où tout le monde porte de beaux vêtements, habite dans de belles maisons remplies d'objets, et possède un style de vie "glamour » :

c'est devenu un standard parmi les minorités, particulièrement les jeunes, qui ont le sentiment d'être inférieurs ou soumis à une distribution inéquitable. Le fossé risque de se creuser, entraînant de sérieuses implications pour l'intégration nationale et la solidarité sociale (Rambo, 1997, p. 24).

La télévision est, par définition, un instrument social créateur de rapports sociaux. Elle joue un « rôle intégrateur » (Mehl, 1992, p. 35). Elle renvoie aux ethnies l'image d'une façon d'être et de vivre ensemble. Les informations, les émissions en direct ou en quasi-direct font que la population voit dans le poste de télévision un miroir, un reflet et en même temps des propositions sur ce qui la rend solidaire d'une collectivité.

Elle ne suscite pas seulement l'assentiment mais, au-delà, l'identification. Identification qui se joue sur l'instant, de part et d'autre du récepteur. Mais identification qui se noue également entre téléspectateurs au terme des transactions qui s'ébauchent le lendemain dans les échanges ordinaires. Important facteur de changement, elle intervient à plusieurs niveaux :

L'éloignement géographique, les notions de distances, sont "virtuellement » abolies grâce à ce média qui permet une meilleure circulation de l'information, une meilleure connaissance des peuples et lieux qui les entourent, tant à un niveau national qu'international.

La notion de temps se modifie également dans l'esprit des montagnards. Tout d'abord parce que l'accès à l'information est plus rapide, mais aussi en raison d'une nouvelle gestion du temps libre, la télévision étant devenu le loisir préféré des minorités.

Le genre de vie en est ainsi changé : les discussions autour de la pipe à eau se font plus rares, les jeux de cartes aussi. Bref, tout un univers collectif où l'échange primait tend à disparaître. Bien entendu, la télévision rassemble encore les gens du village. Mais qu'adviendra-t-il lorsqu'elle sera possédée par tout un chacun? L'individualisme primera alors sur l'esprit communautaire.

D'autant que le système social s'est lui aussi transformé. Le chef n'est pas le seul à posséder la télévision. D'autres, qui se sont lancés dans le commerce et surtout dans le tourisme, gagnent suffisamment d'argent pour pouvoir s'offrir un petit écran, s'élevant ainsi au même niveau social que le chef ou d'autres notables du village. L'argent devient le facteur essentiel de différenciation.

Bien plus que le costume ou l'habitation qui tendent à disparaître, ou du moins, à tenir une place bien moins importante dans l'esprit des montagnards qui, désormais sont influencés par le mode de vie Kinh. Comment, dès lors, se différencier? L'abandon des matériaux de construction, des couleurs et des formes propres aux ethnies minoritaires les amène petit à petit vers le chemin de l'intégration ou de l'assimilation.

La communication devient ainsi une valeur centrale, essentielle de la société :

Mise en scène par les médias, elle fait d'eux des instruments incontournables puisque seul lieu où trouver les informations permettant de décoder les différents univers dans lesquels évolue l'homme moderne (Breton, 1991, p. 243). 

Viêtcommunication, dans le cas qui nous préoccupe ici, se fait de manière implicite, échappe en partie au contrôle du gouvernement, s'effectue essentiellement à travers la possession d'objets occidentalisés dans un contexte d'échanges économiques de biens, amenant à recomposer peu ou prou la société des ethnies basée sur le modèle Viêt.

41 À ce propos, Baudrillard dit que la possession d'objets correspond à une demande objective de signes. De nouvelles hiérarchies sociales pourraient remplacer les anciennes, ce qui signifie que l'organisation sociale des Thaï et des Muong est formée d'un certain nombre de « congères » au sens où l'entend Sorokin. Nous sommes à présent en mesure d'en mettre certaines en lumière :

L'habitation: même si elle reste souvent traditionnelle, bon nombre de modifications ont déjà été apportées (annexes, toiture, etc.), la population aspirant à vivre dans une maison de plain-pied, jugée bien plus pratique ${ }^{16}$. Ainsi, pouvons nous mettre l'accent sur les changements dans la structure de l'habitat: ceux qui sont trop influencés par la télévision ou ceux qui possèdent une moto rêvent d'une maison de plain-pied, jugée bien plus pratique ! Ce serait donc la force matérielle qui serait la source de la force motrice du développement. La moto aurait plus de poids que des millénaires de tradition, les valeurs utilitaires plus d'importance que les valeurs symboliques. C'est la lutte incessante entre modernité et tradition. Mais que va-t-il advenir alors de certaines coutumes comme les coutumes de mariage Thaï ? En effet, chez les Thaï, lorsqu'un garçon est attiré par une jeune fille il va la piquer durant la nuit à travers le plancher du pilotis à l'aide d'un morceau de 
bambou. Si celle-ci est d'accord, elle sort et ils vont passer la nuit ensemble. Que resterait-il de cette coutume si le pilotis disparaissait? Une façon d'être amoureux se modifierait. Comment s'aime-t-on dans une maison de plain-pied? Si ce n'est comme le font... les Viêt.

Les objets occidentalisés qui amènent un nouveau genre de vie basé sur la culture, la recherche du confort et la communication avec le monde extérieur, source même d'intégration. Ils amènent à des changements dans l'organisation de la vie quotidienne : on joue moins aux cartes, on raconte moins d'histoires aux enfants, on regarde plus souvent la télévision qui rythme la journée, au réveil au son des clips vidéo, le soir avec les informations suivies du film ou du dessin animé. Le sens de l'abandon progressif de ces jeux est l'éloignement de la structure collective de ces sociétés. Elles vont vers l'individualité, poussées par des objets que nous connaissons bien et qui ont déjà eu ces mêmes effets sur les sociétés occidentales.

\section{Nous avons également observé :}

La disparition apparente des sorciers dans les villages faciles d'accès. Or, le sorcier était extrêmement important. Relevant du chamanisme, il est l'intercesseur entre le monde des vivants et le monde des morts. Y aurait-il un recul des superstitions? Un vide? Vide qui serait comblé par le médecin ou le vendeur de produits pharmaceutiques sur le marché [la modernité], que l'on appelle plus souvent que le sorcier [la tradition].

Une utilisation plus "pratique» de la géomancie ${ }^{17}$, qui s'adapte en fonction de l'emplacement attribué au futur propriétaire d'une maison, des matériaux qu'il va utiliser, etc. En effet, compte tenu de ce que nous connaissions de la géomancie, nous avons été frappé, lors de notre séjour de 1997, de constater que certaines habitations ne répondaient absolument pas aux critères géomanciques. Nous avons posé la question à trois familles Thaï du village de Mo, dont le terrain était inégal ou entouré de collines. Il nous a été répondu que le géomancien était bien venu faire son travail mais que, compte tenu des difficultés d'obtention d'un terrain, « on s'arrange avec ». Aujourd'hui, la géomancie semble n'être plus qu'un « arrangement avec ». Il devient bien plus important de posséder une maison que de respecter l'ensemble des règles de la géomancie.

Une baisse notable du nombre de jours et de victuailles dans les coutumes de mariages et d'enterrement. Chez les Muong, à propos de celles-ci, par exemple, à l'origine, 12 veillées funèbres doivent être organisées par l'officiant avec un but bien précis pour chaque veillée ${ }^{18}$. Ramenées à 2 ou 3 , elles ne peuvent que passer rapidement ou occulter certains aspects de la nouvelle vie du mort, et modifier ainsi la perception de l'au-delà chez les Muong $^{19}$. Nous ne sommes malheureusement pas en mesure de savoir quels aspects sont occultés puisque nous n'avons pu assister à un enterrement mais tout laisse à penser que les raisons sont économiques. La baisse du nombre de jours et de victuailles est avant tout un problème d'ordre économique, impulsé par Hô Chi Minh lors de la révolution. Il fallait faire des économies pour gagner la guerre. Néanmoins, la guerre ayant cessé, nous pouvons désormais nous interroger sur le sens de cette baisse : Le repas de fête traditionnel possède une signification communautaire importante où les individus d'un clan ou d'une famille réaffirment leurs liens de solidarité à la communauté. Par souci d'économie, mais également pour acheter des «objets individuels ", les ethnies procèdent ainsi à une diminution manifeste de la vie collective. Nous passons ainsi de la contrainte par souci d'économiser - patriotisme oblige - à la restriction par souci d'épargner l'argent en vue de l'achat d'objets modernes. L'esprit communautaire recule devant la montée de l'individualisme.

Mais il y a progressivité de l'intégration des ethnies minoritaires puisque la tradition se maintient malgré tout, les ethnies évoluant ou oscillant constamment entre tradition et modernité. Le degré d'évolution des ethnies donne cependant le sentiment d'être respecté. 

« emboîtent » en quelque sorte, pour reprendre le terme de Lévi-Strauss ${ }^{20}$, faisant de la Nation viêtnamienne une entité capable de s'insérer dans un ensemble plus vaste : celui de l'Asie du sud-est. L'achat d'une télévision, par exemple, joue un rôle fondamental puisqu'elle donne à voir, à travers des émissions diverses (soaps, clips vidéo...), ce qui est Viêt, mais aussi un monde qui s'élargit à l'Asie du sud-est ou à l'Occident (films en provenance de Hong-Kong, dessins animés américains...).

L'insertion s'effectue en partie à travers les échanges économiques auxquels les ethnies minoritaires participent à leur modeste niveau.

\section{La Viêtcommunication}

\section{Synthèse}

La Viêtcommunication est un processus en vue d'une assimilation qui se fonde sur le rapport entre la montagne et la plaine, la plaine étant le modèle de développement vers lequel tend le montagnard. En cela :

C'est un processus typiquement viêtnamien d'intégration.

Ce processus ébranle les cultures des minorités là où elles sont ductiles - leurs congères - et par voie de conséquence, remet en question leurs systèmes.

Il s'effectue par plusieurs biais : l'un passe par le contrôle du Parti, qui s'efforce de mettre en place toute une série de politiques (hygiène, économique, éducative et culturelle) depuis les années 1970. Dans ce cas, l'intégration des ethnies minoritaires à l'État-Nation viêtnamien a une portée idéologique; l'autre, qui s'insinue depuis 1986 et l'ouverture du pays à l'économie de marché, grâce à toute une série d'échanges et l'apparition d'objets occidentalisés qui vont modifier le genre de vie des ethnies et s'attaquer de manière

beaucoup plus rapide et efficace à certaines congères (costume, habitation, etc.). L'intégration se fait alors par un second biais échappant dans une large mesure au Parti-État. C'est une intégration par l'économie.

Enfin, c'est un processus dans lequel les écarts perdurent. Autrement dit, le modèle de référence Viêt, est un modèle déjà dépassé dès l'instant où les viêtnamiens des plaines et des villes, à leur tour, s'alignent sur un modèle en perpétuelle évolution, le « modèle occidental » aux contours des plus larges.

\section{Accroissement}

N'étant pas retournée au Viêt-Nam depuis 1997, il nous est impossible d'apporter des données chiffrées sur l'accroissement de la Viêtcommunication. Cependant, l'État ayant procédé à l'aménagement routier des régions montagneuses en l'an 2000, nous pouvons supposer qu'elle touche désormais des populations plus reculées par les échanges qui peuvent dorénavant s'y opérer de manière relativement aisée. Introduction d'objets occidentalisés, tourisme, modification de l'habitation, etc., ne seraient alors plus l'apanage de la seule région de Hoa Binh ${ }^{21}$. 


\section{BIBLIOGRAPHIE}

Le lecteur constatera l'ancienneté de la plupart des références ci-dessous. Qu'il ne s'en étonne pas : nous avons utilisé :

- d'une part, des ouvrages considérés comme références par les spécialistes du Viêt-Nam ;

- d'autre part, des écrits d'obédience ethnologique (de référence eux aussi), sur lesquels nous nous sommes basé pour noter les écarts avec le Viêt-Nam actuel, aucune monographie récente n'ayant été publiée à notre connaissance sur les Thaï ou les Muong ;

- enfin, des textes éclairant les politiques d'intégration du Parti, datant donc des années 70.

\section{Ouvrages concernant le Viêt-Nam :}

Agence de Cooperation Culturelle et Technique, (1984), Alphabétisation et éducation des adultes en République Socialiste du Viêt-Nam, Paris, collection alpha, $\mathrm{n}^{\circ} 9$.

Basilico, S., (2001), La « Viêtcommunication » ou le processus d'insertion des ethnies Thaï et Muong dans l'État-Nation viêtnamien en développement, Mémoire de thèse d'anthropologie.

Basilico, S., (2003), Mondialisation et échanges au Viêt-Nam, Paris, L'Harmattan.

Bezacier, L., (1955), L'art viêtnamien, Paris, Union Française.

Cadiere, L., (1957), Croyances et pratiques religieuses des viêtnamiens, Paris, EFEO.

Cuisinier, J., (1948), Les Muong, Paris, Institut d'ethnologie.

Diguet, E., (1908), Les montagnards du Tonkin, Paris, Chalamell.

Fourniau, P., (1985), « L'enseignement » in : Viêt-Nam, l'Histoire, la terre, les hommes, Paris, L'Harmattan.

Gourou, P., (1936), Les paysans du delta tonkinois, Paris, Mouton et Lahaye.

Hô, Chi Minh, (1994), Écrits (1920-1969), Hanoï, The Gioi.

Huard, P. et Durand, M., (1954), Connaissance du Viêtnam, Hanoï, EFEO.

Len, Hao, (1971), «Études et recherches ethnologiques au nord Viêt-Nam », in : Études viêtnamiennes, $\mathrm{n}^{\circ} 32$.

Nguyen, Tu Chi, (1997), La cosmologie Muong, Paris, L'Harmattan.

Nguyen, Tung, (1985), Les viêtnamiens et le monde surnaturel, Paris, Lidis.

Nguyen, Van Huy, (1997), Les politiques d'intégration des montagnards des hauts plateaux du centre du Viêtnam (des origines à 1958), Thèse de Doctorat, Université de Paris VII.

Nhat, Hung, (1967), « L'enseignement au service des minorités nationales », in : Études viêtnamiennes, $\mathrm{n}^{\circ} 15$.

Quang, Canh, (1967), « Les transformations économiques dans les régions montagneuses », in : Études viêtnamiennes, $n^{\circ} 15$. 
Rambo, A. T., (1997), Development trends in Vietnam's northern mountain region, Center for natural resources and environmental studies, Hanoï, Vietnam National University, National Political Publishing House.

Rousset, P., (1978), Communisme et nationalisme Viêtnamien, Paris, Galilée.

Roville, G. et Zimbardo, X., (1994), Viêtnam, au pays des routes contraires, Paris, Peuples du Monde.

\section{Ouvrages concernant la communication :}

Baudrillard, J., (1991), La Société de consommation, Paris, Folio Essais.

Baudrillard, J., (1990), Le système des objets, Paris, Folio Essais.

Breton, P., (1991), « L'idéologie de la communication et l'emprise des médias », in : Charon J.-M. (éd.), L'état des médias, Médiaspouvoirs, Paris, La découverte.

Debray, R., (1992), Vie et mort de l'image. Une histoire du regard en occident, Paris, Gallimard.

Hall, E. T., (1987), La dimension cachée, Paris, Seuil.

Hall, E. T., (1986), Le langage silencieux, Paris, Seuil.

Mehl, D., (1992), La fenêtre et le miroir. La télévision et ses programmes, Paris, Payot.

Michel, H., (1989), La télévision en France et dans le monde, Paris, P.U.F.

Morin, E., (1977), La méthode 1 - La nature de la nature, Paris, Point Seuil.

Morin, E., (1980), La méthode 2 - La vie de la vie, Paris, Point Seuil.

Morin, E., (1983), La méthode 4 - Les idées, Paris, Point Seuil.

Morin, E., (1991), Introduction à la pensée complexe, Paris, ESF.

Proulx, S., (1991), « Deux grands courants dans les recherches et théories sur les médias », in : Charon J.-M. (éd.), L'état des médias, Médiaspouvoirs, Paris, La découverte.

Popper, K., (1995), La télévision, un danger pour la démocratie, Paris, Anatolia.

Sfez, L., (1992), La communication, Paris, P.U.F.

Sfez, L., (1993), Dictionnaire critique de la communication, Paris, P.U.F., (2 vol.).

Viches, L., (1995), La télévision dans la vie quotidienne, Paris, Apogée.

Winkin, Y., (1996), Anthropologie de la communication, De Boeck Université.

Winkin, Y., (1980), La nouvelle communication, Paris, Seuil.

Wolton, D., (1990), Éloge du grand public. Une théorie critique de la télévision, Paris, Flammarion.

Wolton, D., (1997), Penser la communication, Paris, Flammarion.

\section{Ouvrages anthropologiques :}

Sorokin, P. A., (1947), Society, culture and personality, New-York.

Levi-Strauss, P., (1952), Race et Histoire, Paris, UNESCO. 


\section{NOTES}

1. Littéralement, « homme de la capitale ».

2. Nous n'entrerons pas dans le détail de ces coutumes et croyances, puisqu'il ne s'agit pas d'une publication à caractère ethnologique. Sans entrer dans le détail, disons que les minorités possèdent une culture propre : leur habitation est sur pilotis (contrairement aux Viêt dont la maison est de plain pied), leurs costumes traditionnels sont très colorés (à la différence du costume sombre des Viêt), leur langue est fort différente du Viêt (bien que le viêtnamien soit devenu langue officielle du pays), leur organisation sociale traditionnelle est basée sur le féodalisme. Leurs coutumes d'enterrement ou de mariage sont également très différentes. Voir à ce sujet certaines notes en bas de page pour plus d'information.

3. Nous appellerons produits occidentalisés les produits à forte connotation occidentale (c'est-àdire qui n'ont aucune fonction traditionnelle au Viêt-Nam, par exemple télévision mais aussi cigarette, jean, casquette, etc...) mais qui sont fabriqués en Asie, généralement Chine ou ViêtNam.

4. Cette région a été choisie pour 2 raisons ; 1 . Avant toute chose, cette région constitue l'une des plus riches d'un point de vue inter-ethnique puisque de nombreuses ethnies y cohabitent (Viêt, Hmong, Dao, Muong, Thai, Tay). Elle est donc fort intéressante pour une étude portant sur les échanges inter-ethniques ; 2. Par ailleurs, il s'agit d'une des régions les plus proches de la capitale (environ 4 heures de route en voiture). De ce fait, nous supposons qu'il est plus simple d'y introduire toutes sortes de produits susceptibles de favoriser une certaine évolution chez les montagnards, à savoir des produits que nous qualifierons d'occidentalisés, parce qu'ils véhiculent tout un mode de vie qui se distingue largement de la vie traditionnelle Viêtnamienne. L'ennui de ce choix favorable, c'est qu'il risque de ne pas être significatif de la réalité. Nous sommes conscient de cette difficulté. Nous ne pensons pas cependant qu'il puisse hypothéquer, encore moins remettre en question la validité de notre enquête. Il va sans dire que nos résultats ne valent que pour cette période, allant de 1995 à 1997, et cette région.

5. Phrase bien connue prononcée par le Résident Supérieur P. Pasquier.

6. Chez les Muong le génie du foyer est un dieu multiple ou un dieu commandant à d'autres dieux mineurs. Son culte se confond avec celui des génies protecteurs. Il est bien sûr le protecteur d'une maison et de la famille qui l'habite, mais aussi témoin de tout ce qui s'y passe. Il fait en effet son rapport sur les bonnes ou mauvaises actions des habitants chaque année au roi du ciel.

7. Universalis.

8. P. A. Sorokin fait la distinction dans Society, culture and personality, New-York, 1947, de deux notions formant la culture ; 1 . Les systèmes qui sont des ensembles globaux et homogènes ou des ensembles particuliers de sens et de valeurs ; 2 . Les congères qui sont des éléments contingents. Tout ce qui relève des emprunts, des germes de nouveauté, c'est-à-dire tout ce qui est extérieur au fond culturel, relève des congères.

9. C'est le doi moi ou « renouveau économique » impulsé par le Parti depuis 1986 et qui touche les minorités depuis 1995.

10. Un montagnard qui s'adonne au tourisme gagne en moyenne 1 euro par jour. Une télévision couleur vaut environ 152 euros, un magnétoscope, un réfrigérateur ou une chaîne hifi 304 euros. Mais il est possible d'acheter d'occasion : une télévision couleur vaudra alors environ 76 euros, et il faut compter entre 122 et 152 euros pour une chaîne hi-fi ou un magnétoscope. Vide tableau cidessous.

11. Que nous avons évoquée un peu plus haut.

12. Soap: Téléfilm d'origine américaine, autrefois sponsorisé par des marques de lessive (d'où son nom). 
13. Ao Dai : Costume traditionnel viêtnamien.

14. Terrain SB septembre 1997.

15. Étude sur le terrain réalisée, grâce à 2 séjours d'un mois en 1995 puis 1997, à l'aide d'un questionnaire et d'une grille d'analyse de l'habitat visant à déterminer si les modifications que nous avions remarquées en 1995 (en comparaison avec des écrits plus anciens) se propageaient, ce qu'elles pouvaient engendrer du point de vue culturel, institutionnel et économique. Étude réalisée dans la région de Hoa Binh, choisie pour son extrême diversité ethnique et pour sa proximité géographique d'Hanoï, favorisant les échanges commerciaux.

16. Ce qui ne signifie pas pour autant que les ethnies minoritaires sont prêtes à abandonner leur organisation familiale.

17. Géomancie : Étude de l'implantation d'une habitation en fonction de critères géographiques correspondant au souffle vital afin de porter chance à la famille.

18. Les 12 veillées funèbres se répartissent ainsi chez les Muong; 1 . Oraison sur la naissance de la terre et de l'eau ; 2 . Oraison sur « la chasse du tigre et la coupe de l'arbre »; 3. Appel des ancêtres du mort pour préserver les vivants; 4 . Appel aux ancêtres du ciel; 5. Plaidoirie pour que les animaux tués par le défunt ne réclament pas vengeance au roi du ciel ; 6 . Le génie-patron guide le mort nouveau parmi les anciens morts qui lui procureront l'argent nécessaire à ses premières dépenses dans son nouveau monde; 7. Demande d'une âme; 8. Descente du génie-patron avec l'âme du mort; 9. Promenade de l'âme; 10. Enseignement sur le nouveau comportement à adopter par le mort ; 11. et 12. Nuits d'offrandes et de lamentations.

19. La cosmologie Muong est composée d'un univers à 3 niveaux répartis sur un axe vertical : Au milieu, c'est le Muong Pua («terroir plat ») représentant le monde des vivants. Ses frontières peuvent se distendre et inclure des régions étrangères aux «terroirs » Muong, notamment Ke Cho (" enfantement de la terre et de l'eau ») qui s'ouvre sur un système de mythes d'origine, lequel sert de point de départ à des événements qu'on peut qualifier de pseudo-historiques (apparition et consolidation d'un grand domaine unifié et dont les limites englobent aussi le pays des «Kon Tao», Viêt et celui des «Kon Ju», Thai) ; le Muong Kloi («terroir du ciel») est le domaine où s'exerce l'autorité du roi du ciel, entouré de ses acolytes répondant au titre de Kem et qui sont des notables; le monde du sous sol, qui n'est pas un enfer, mais un monde reproduisant le "terroir plat» en miniature, rempli de petits hommes qui se nourrissent de terre. De tous temps, il a existé une possibilité de communication entre ces trois mondes par le biais d'un goulot.

20. Pour Lévi-Strauss (Race et Histoire, Paris, UNESCO, 1952), une culture « s'affirme moins en ellemême qu'en opposition à d'autres". Si bien que lorsqu'on parle de culture, il faut aussi parler d'emboîtements des cultures les unes dans les autres.

21. Les politiques d'intégration du Parti ayant, quant-à elles, eu lieu dans tout le pays mais avec, bien entendu, des possibilités de vérifications moindres dans les zones les plus reculées. Par exemple, la politique de natalité autorise 2 enfants par couple. Mais chez les minorités, ils sont bien plus nombreux...

\section{RÉSUMÉS}

La «Viêtcommunication", processus d'insertion des ethnies Thaï et Muong à l'État-Nation viêtnamien à travers l'échange d'objets - essentiellement occidentaux - que l'on retrouve désormais au sein des minorités, s'effectue par plusieurs biais : l'intégration idéologique passant 
par le contrôle du Parti qui s'efforce depuis les années 70, de mettre en place toute une série de politiques, l'intégration économique qui s'insinue depuis 1986 et l'ouverture du pays à l'économie de marché, grâce aux échanges et à l'apparition d'objets occidentalisés qui modifient le genre de vie des ethnies et s'attaque à certains éléments de leur culture.

Viet-communication, or the process of inserting Thai and Muong people into the Vietnamese nation, takes place through exchanged objects, which are now held in the houses of members of minorities, and acts through two mechanisms: ideological integration, with the Communist Party using various policies since the 1970s, and economic integration since 1986 through exchanges and the emergence of Westernized objects that modify some elements of the minorities' cultures.

\section{INDEX}

Mots-clés : ethnologie, communication linguistique, état, intégration, Vietnam

Keywords : ethnology, linguistic communication, state, integration, Vietnam

\section{AUTEUR}

\section{SANDRINE BASILICO}

Lamic - Université de Nice

Sandrine Basilico est Docteur en Anthropologie de la Communication Sociale, chercheur au LAMIC (Université de Nice). Sa thématique de recherche - la mondialisation - s'effectue sur l'aire asiatique comme en témoignent ses différentes publications, dont : Mondialisation et intégration des minorités ethniques au Viêt-Nam, L'Harmattan, 2003. « Les objets occidentalisés et la télévision vecteurs du développement du sentiment d'appartenance des ethnies Thaï et Muong », Terminal, L'Harmattan, 2002. 